\title{
Interacting with objects compresses environmental representations in spatial memory
}

\author{
Laura E. Thomas • Christopher C. Davoli • \\ James R. Brockmole
}

Published online: 16 October 2012

(C) Psychonomic Society, Inc. 2012

\begin{abstract}
People perceive individual objects as being closer when they have the ability to interact with the objects than when they do not. We asked how interaction with multiple objects impacts representations of the environment. Participants studied multiple-object layouts, by manually exploring or simply observing each object, and then drew a scaled version of the environment (Exp. 1) or reconstructed a copy of the environment and its boundaries (Exp. 2) from memory. The participants who interacted with multiple objects remembered these objects as being closer together and reconstructed smaller environment boundaries than did the participants who looked without touching. These findings provide evidence that action-based perceptual distortions endure in memory over a moving observer's multiple interactions, compressing not only representations between touched objects, but also untouched environmental boundaries.
\end{abstract}

Keywords Spatial memory · Environments · Interaction · Grasping · Action-specific perception · Embodied cognition

Intuition may suggest that we perceive and represent the world through objective, true-to-life, and behaviorally independent representations, but a growing body of research instead shows that perceptions are embodied, changing as a function of one's intentions and capabilities, and of the costs for action (Proffitt, 2006). For example, athletes who are playing well recall larger goals and slower ball dynamics (e.g., Witt \& Dorsch, 2009; Witt \& Sugovic, 2010), people

L. E. Thomas $(\bowtie)$

Department of Psychology, North Dakota State University,

NDSU Dept. 2765, P.O. Box 6050, Fargo, ND 58108-6050, USA

e-mail: laura.e.thomas@ndsu.edu

C. C. Davoli · J. R. Brockmole

University of Notre Dame,

Notre Dame, IN, USA in chronic pain perceive farther distances between objects (Witt et al., 2009), and people with stronger grip strength judge their hands to be larger (Linkenauger, Witt, Bakdash, Stefanucci, \& Proffitt, 2009). Interactions with objects also carry with them distortions of perceived space: An object that is out of arm's reach looks closer to a person who can interact with it using a tool (e.g., Davoli, Brockmole, \& Witt, 2012; Witt \& Proffitt, 2008; Witt, Proffitt, \& Epstein, 2005). Collectively, results like these have given rise to the actionspecific account of perception (Witt, 2011), which posits that optical information is scaled relative to an observer's ability to act on objects in the visual field, whether that ability is derived from native skill or tool use.

One constraint on the action-specific account of perception is its focus on situations in which an observer interacts with a single object (e.g., a ball). In the real world, interactions are rarely so limited. Typically, people maneuver within environments containing several potentially task-relevant objects that are distributed throughout space, such as when making dinner in a kitchen or working in a garden. While interaction with a single object shapes how a person perceives that object in relation to him- or herself, it is unclear whether interaction with multiple objects - each viewed from a different vantage point-impacts perception and representation of the environment as a whole. In this article, we investigate this issue within the context of remembered distances.

A variety of methodological paradigms have shown that interacting with objects causes observers to underestimate body-to-object distances when making real-time judgments about the distance of single objects from a fixed point of observation (Bloesch, Davoli, Roth, Brockmole, \& Abrams, 2012; Davoli et al., 2012; Witt \& Proffitt, 2008; Witt et al., 2005). Following interaction with an object, observers also underestimate their remembered body-to-object distance (Davoli et al., 2012). This kind of underestimation in memory is consistent with the spatial compression that occurs during the moment of interaction, and it likely reflects the 
encoding of that compressed perception of viewer-to-object distance into memory (Davoli et al. 2012). But, would such compression be incorporated into an observer's holistic representation of an environment in which he or she moved from place to place to handle multiple objects? When observers experience a navigable space from a small number of different viewpoints, they create viewpoint-dependent representations in memory (i.e., Shelton \& McNamara, 1997). Therefore, it is possible that compression of space would be viewpoint dependent, relative to the position of the self during the moment of interaction. In this case, compression might only manifest upon probing that particular viewer-to-object relationship, but not when probing a moving observer's representation of a multiobject environment. If verified, this result would suggest that perceptual changes based on action are spatially specific.

Another possibility is that viewers hold action-based perceptual distortions in memory across multiple interactions. Observers are able to keep track of object positions relative to the self across movements (Simons \& Wang, 1998; Wang \& Simons, 1999), and the maintenance of such egocentric representations may allow previously distorted viewer-toobject positions to endure in spatial representations of a multiobject environment. In this case, observers who handle multiple objects in an area should remember smaller object-toobject distances. If multiple interactions cause spatial compression, a corollary question concerns the scope of this distortion: Is compression limited to objects that receive handling (local distortion), or does it transfer to the parameters (i.e., untouched boundaries) of the entire environment (global distortion)?

In the present study, we examined how interaction influences observers' representations of the local and global spatial details of multiobject environments. We first asked participants to walk through a room containing various objects. Participants either picked up each object or viewed objects without touching them. Later, each participant recreated the environment. Experiment 1 followed previous research by asking participants to draw a map of the observed space (e.g., Davoli et al., 2012). In Experiment 2, we moved beyond scaled representations by asking participants to physically reconstruct the studied environment. If interactions with multiple objects can simultaneously influence multiple representations of interobject spatial relations, then we would expect the maps and reconstructions to differ according to whether or not observers handled the objects. Consistent findings obtained across two different dependent measures would provide converging evidence that our observations were not dependent on the testing paradigm and would confirm the validity of assessing representations of real-world space with scaled testing procedures. In Experiment 1, we focused our investigation on whether action-dependent compression would survive across a moving observer's multiple interactions and would translate into smaller remembered object-to-object distances. After finding evidence for spatial compression in Experiment 1, we turned our attention to the locus of the compression effect. In Experiment 2, observers recreated previously studied object locations and the physical boundaries of the environment, allowing us to independently measure spatial distortions of the environment's local and global properties.

\section{Experiment 1}

In Experiment 1, we examined the impact of interactionsdefined here as taking hold of an object with the hands - on incidental memory representations of a multiobject environment. Participants studied the objects in a room for their details but were later probed on the objects' locations. We compared the representations of participants who manually inspected each object with those who looked at the objects without touching, by either inspecting every object from a unique vantage point or studying the room from one fixed viewing location.

\section{Method}

Participants A group of 62 North Dakota State University undergraduates participated for course credit.

Materials, apparatus, procedure, and design Six stuffed animals sat on the floor of a study room (Fig. 1). The participants were instructed to examine each object and to memorize its details for a future memory test. Participants were not told that they had to remember the locations of the objects. The experimenter prompted participants to study objects one by one, naming an animal every $15 \mathrm{~s}$ in a different random order for each participant. Participants in the manual condition $(n=20)$ walked to each object, picked it up, and held it until the next animal was named (at which time they placed the object back where it had been on the floor). Participants in the walking condition $(n=22)$ walked to each object, but simply stood in front of the object without touching it. Participants in the stationary condition $(n=20)$ remained standing in the study room's doorway throughout the study phase and were instructed to look at objects as each was named. Following this study phase, participants entered a separate room and received a blank sheet of paper. The experimenter asked them to mark the remembered location of each object with a dot and a label. The object names were provided to the participants. The experimenter gave no additional instruction about how to map the study room space onto the blank sheet. After the test phase, participants filled out a posttest questionnaire that asked what they thought the purpose of the study was and what they predicted the results of the study would be. 


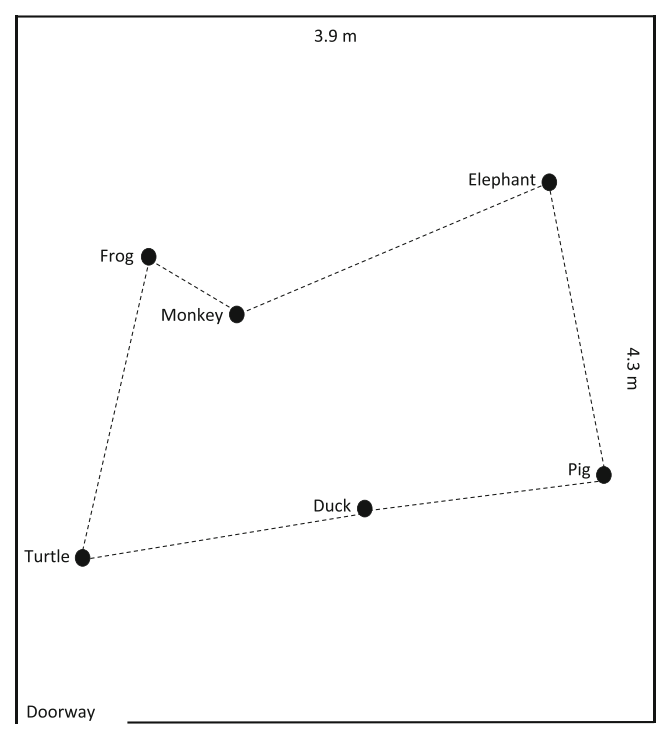

Fig. 1 Layout of objects in the study room for Experiment 1

\section{Results and discussion}

To quantify spatial memory representations, we took the completed maps and coded each object's dot in terms of $x$ and $y$-coordinates in centimeters. Using these coordinates, we generated a six-sided polygon for each participant by treating the object dot locations as vertices (an example using the actual object positions is illustrated in Fig. 1). We then calculated the area of this polygon as well as the distance between every possible set of object pairs. We excluded participants from our analyses if they created maps that did not correctly portray the relative spatial relationships between objects (i.e., the objects located at adjacent vertices of the resulting polygon did not match those shown in Fig. 1). This trim minimized the possibility that faulty memory for the relative positions of objects could contaminate our assessment of memory for their spatial separation. This trim left 17 observers in each condition. ${ }^{1}$

Our interaction manipulation impacted both the areas of the object configurations $[F(2,50)=3.75, p=.031$; see Fig. 2a] and the average object-pair distances $[F(2,50)=$ 4.66, $p=.014$; see Fig. 2b]. Post-hoc least significant difference analyses indicated that participants in the manual condition drew object configurations with smaller areas than did participants in both the walking $(p=.035)$ and stationary $(p=.015)$ conditions. Those in the manual condition further reported objects as being closer together, on average, than did participants in both the walking $(p=.027)$ and stationary $(p=.006)$ conditions. Polygon areas did not differ between the stationary and walking conditions $(p=.72)$, nor did average object-pair distances $(p=.54)$.

\footnotetext{
${ }^{1}$ We obtained consistent results if we retained the excluded participants in the analyses.
}

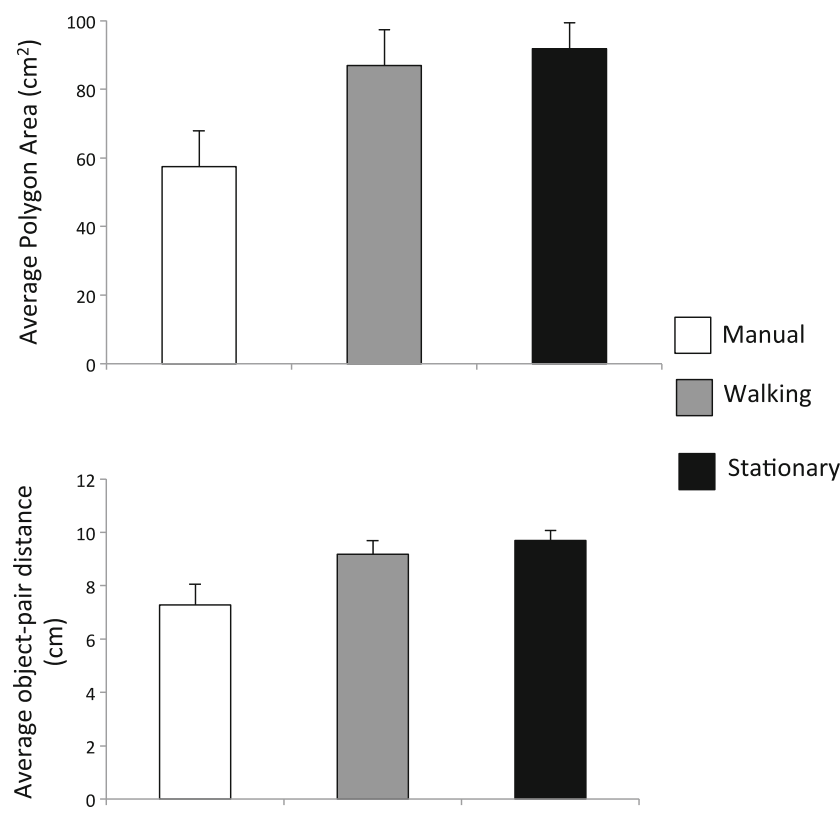

Fig. 2 Average areas and object-pair distances for the drawings created in Experiment 1

Participants who handled objects remembered smaller object-to-object distances, and subsequently drew a more compressed representation of the object array than did either participants who walked around the objects without touching anything or participants who remained stationary. This finding provides the first evidence that interaction-driven distortions of space endure across a moving observer's multiple actions, compressing interobject representations. Strikingly, even though there were substantial viewing differences between the walking and stationary conditions, participants in these conditions drew maps that were similar in terms of spatial scale, ruling out the possibility that physical effort or proximity to stimuli leads to compression independently of physical interaction.

Recently, it has been argued that certain demonstrations of action-specific perception (e.g., Bhalla \& Proffitt, 1999) do not reflect perceptual distortions, but rather the participants' ability to ascertain the experimenters' hypotheses and to alter their responses accordingly (see, e.g., Durgin et al., 2009). Could demand characteristics explain the results that we obtained in Experiment 1? Analysis of the posttest questionnaires showed that no participant mentioned anything about compression of space, nor did they guess that we were interested in the effects of physical interaction per se. Instead, most participants across all three conditions assumed that the purpose of the experiment was to investigate how well they remembered locations when they were not explicitly instructed to encode this information. Thus, it is unlikely that the compression effects that we found were driven by demand characteristics. Instead, our data point to the conclusion that physically interacting with 
an environment leads people to remember its objects as being closer together.

\section{Experiment 2}

The results of Experiment 1 suggest that spatial memory representations are more compressed when observers handle several studied objects than when they do not. In Experiment 2, we tested the robustness of interactiondriven distortions of space, asking participants to reconstruct a full-sized replica of a previously studied environment after either manually or visually inspecting its objects. If spatial compression is a fundamental property of how moving observers represent an environment following interaction, then participants who manually inspect an area's objects should reconstruct full-sized replicas of that environment that are smaller than those of participants who merely engage in visual inspection. In addition, if interaction-driven distortions shape global representations of the environment as a whole, then participants who manually inspect objects should also experience memory compression for the untouched boundaries of the environment.

\section{Method}

Participants A group of 20 University of Notre Dame undergraduates participated for course credit.

Materials, apparatus, procedure, and design We conducted the experiment inside a $6,400 \mathrm{~m}^{2}$ geodesic dome (Fig. 3a). On one side of the dome, we constructed the study area, a 3.96-m square space marked by athletic cones at each corner (Fig. 3b). Ten objects were placed within this space at locations and orientations held constant across participants. The participants' task was to walk to each object and memorize, in $10 \mathrm{~s}$, its visual details. As in Experiment 1, participants were not explicitly instructed to memorize the location of the objects. The experimenter prompted each participant to proceed to the next object in a unique, randomly determined order. Participants in the manual condition $(n=10)$ picked up the objects to study them and placed them back where they had been on the floor when it was time to study the next object. Participants in the visual condition $(n=10)$ were told to analyze the objects from any angle, but that they were not to touch the objects. ${ }^{2}$ Participants were not instructed to study any of the cones.

After the study phase, participants were led to the test area on the other side of the dome for a reconstruction

\footnotetext{
${ }^{2}$ Due to the lack of differences between the walking and stationary conditions in Experiment 1, we used one, free-form visual inspection condition in Experiment 2.
}

phase. The participants were asked to reconstruct, from memory, a full-sized replica of the study area by placing duplicate cones and objects on the floor in their remembered locations (Fig. 3c). One "corner" of the environment was provided by a reference cone placed at the intersection of two guidelines on the floor, so as to ensure the presence of a 90-deg angle in the reconstruction. These guidelines extended well beyond the dimensions of the study area (Fig. 3c). A curtain separated the test and study areas, so that participants in the study area could not see the test area, and vice versa (Fig. 3a).

\section{Results and discussion}

We calculated each reconstructed area's width and depth by measuring the distance between the reference cone and each of the adjacent cones. We also measured the distance between every combination of object pairs, yielding an average object-pair distance.

As compared to the participants in the visual condition, participants in the manual condition reconstructed areas with smaller average object-pair distances (1.87 vs. $2.36 \mathrm{~m} ; t(18)=4.32, p<.001)$, smaller widths (3.56 vs. $4.35 \mathrm{~m} ; t(18)=2.78, p=.012)$, smaller depth (3.64 vs. $4.33 \mathrm{~m} ; t(18)=2.84, p=.011)$, and smaller area $(13.00$ vs. $\left.19.08 \mathrm{~m}^{2} ; t(18)=3.09, p=.006\right)$. These results replicate the core findings of Experiment 1, suggesting that spatial compression of object-to-object distances is a fundamental property of how observers represent environments following interactions. Interestingly, this compression of the overall object configuration was also mirrored in a compression of the space framing the objects: Although participants in the manual condition never touched the cones representing the boundaries of the study area, they nevertheless reconstructed areas in which the cones were closer together than did participants in the visual condition. Handling the objects in an environment therefore not only compresses representations of the objects' configurations, but also leads participants to remember the entire space containing the objects as smaller.

Before proceeding, we note that although we have followed previous research in referring to the differences in spatial memory between manual and visual conditions as interaction-driven compression (e.g., Bloesch et al., 2012; Davoli et al., 2012; Witt \& Proffitt, 2008; Witt et al., 2005), it is conceptually possible that noninteractive study instead leads to a relative expansion of space in memory. While the scaled maps of Experiment 1 did not allow us to directly address this possibility, we can address this alternative account in Experiment 2. The mean width and depth reproduced by the participants in the manual condition were both significantly smaller than the veridical means of the study 

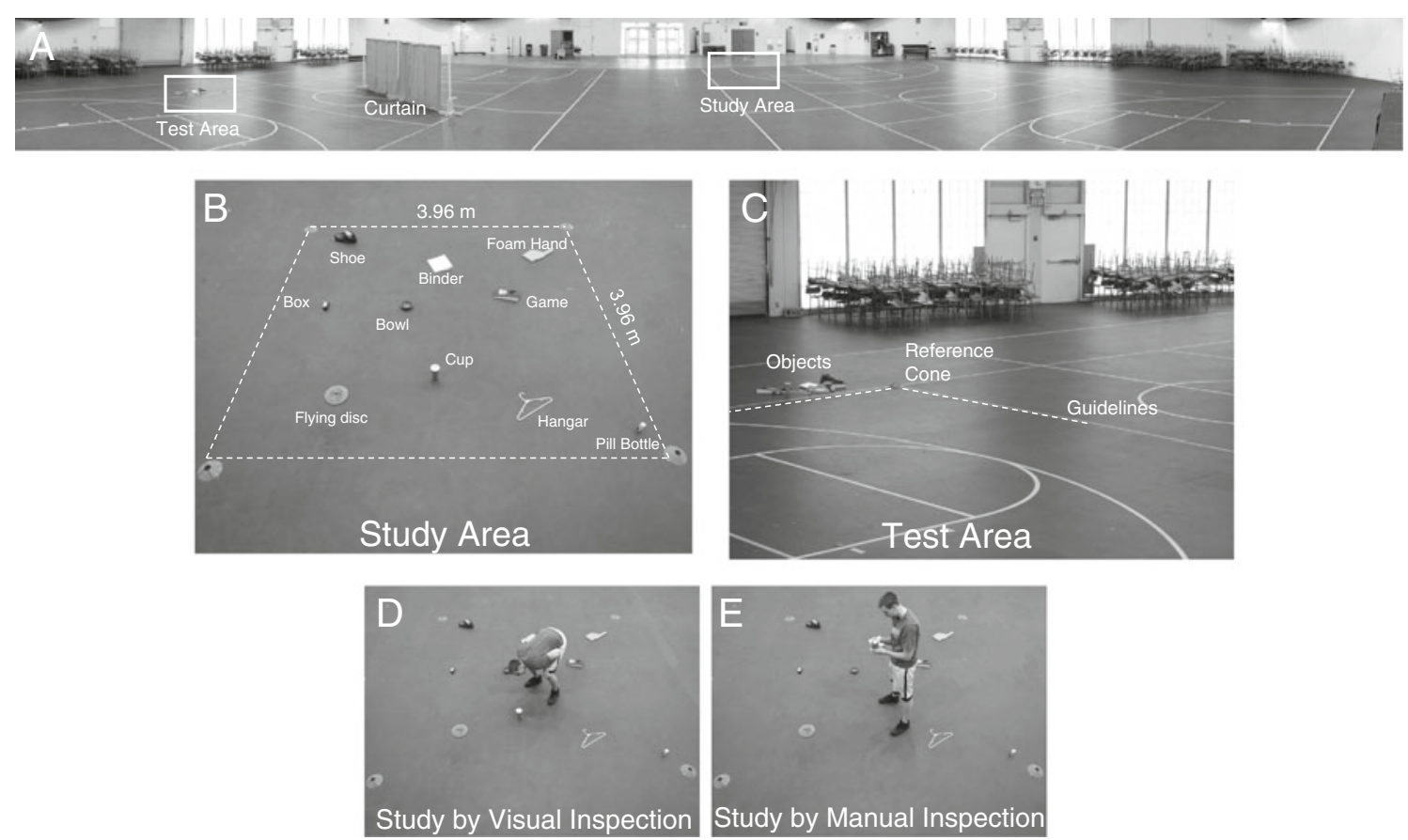

Fig. 3 a The overall testing environment used in Experiment 2. b,c The study and test areas. d,e The testing conditions

area $[t(9)=2.42, p=.039$, and $t(9)=3.12, p=.012$, respectively]. In contrast, the reconstructed environments made by participants in the visual condition did not differ from the veridical size of the study area in either width or depth $[t \mathrm{~s}(9)<1.68, p \mathrm{~s}>.127]$. These results support the notion that handling objects specifically leads to perceptual compression of perceived space.

\section{General discussion}

In two experiments, we have shown that moving through an environment and interacting with multiple objects leads to compressed memory representations of both interobject distances and environmental boundaries. Participants who handled objects drew scaled maps that were more compact and reconstructed smaller environments than did participants who only looked at these objects. In fact, participants who interacted with objects produced an area that was smaller than the space that they studied, whereas participants who did not handle objects reconstructed environments that were spatially similar to the studied area. Whether measured through map making or physical reconstruction of the environment, the extents of compression were remarkably similar, with participants in the manual conditions drawing or reconstructing areas three quarters the size of those in the other conditions.

Why did participants who handled the objects that they studied tend to remember smaller environments than did participants who studied the objects without touching? Our data rule out several possible explanations that are often offered as alternatives to action-modulated perception. For example, we can reject explanations based on variations between conditions in terms of physical exertion or optic flow, either of which could provide distance cues. In Experiment 1, spatial memory was equivalent among stationary individuals who studied objects from a single point in space and those who walked from object to object without picking them up. We only observed compression when observers handled the objects. Visual changes that may be correlated with spatial proximity to objects also seem to be an unlikely explanation. In the manual conditions, participants picked up objects, holding them close, whereas in the other conditions, objects remained on the floor and were on average farther away from participants. Critically, however, participants who stood in the doorway, viewing objects from the farthest average distance, drew maps that were spatially similar to those of participants who walked close to objects but did not handle them, suggesting that proximity or viewpoint differences across conditions cannot solely explain the compression effects that we observed. We can also rule out the possibility that variations in attentional effort or functional field of view account for our findings. If anything, stationary participants exerted more effort and obtained a broader field of view by virtue of studying the objects from afar, but compression was not observed within this group. Finally, the compression effects observed in this study do not seem to be driven by peculiarities in the experimental design, as observers were unable to predict our hypotheses and the effects were robust across different levels of 
memory load (six vs. ten objects) and different types of reporting (maps vs. reconstructions).

Because differences in physical exertion, visual information, and demand characteristics cannot explain the compression effects that we observed, we instead appeal to the notion that physical handling of the objects specifically influenced memory representations. Under this view, our findings have major implications for embodied and action-specific accounts of perception. First, the effects of action on perception endure across multiple viewpoints. Previous demonstrations of action-specific perception had involved interaction with a single object viewed from a single perspective and tests that probed this single viewer-to-object relationship. Because observers viewed multiple objects, each from a different vantage point, the viewer-to-object relationships defined at the moment of interaction were constantly invalidated. Nevertheless, holistic memory representations following interaction were compressed, indicating that the effects of action on perception survived across multiple interactions. Second, action-specific effects create distortions of an environment's global properties. Until now, action-modulated perception has only been studied in terms of local changes in the spatiotemporal representation of the acted-upon object. Here, observers not only represented compressed interobject relationships, they also represented compressed environmental parameters, such as spatial boundaries that were never the target of interaction. This indicates that perceptual distortions resulting from observer-object interactions are applied to the entire visual field. It is possible that these effects are adaptive. For example, observers may scale objects that have been successfully handled as closer (i.e., more reachable) to encourage the encoding of such objects as ones that may afford interaction in the future. By those terms, the successful handling of multiple objects in an environment, as we reported here, appears to give way to the scaling of the entire environment as being more easily maneuverable in terms of total distance.

The experiments we presented here also help bridge the embodied- and spatial-cognition literatures. In both experiments, the participants were required to create an integrated representation of a novel environment in memory. Knowing the locations of objects seems to depend on both a transient system that is chiefly supported by perceptual processes (e.g., Wang \& Spelke, 2000) and an enduring system that is supported primarily by long-term memory (e.g., Mou, McNamara, Valiquette, \& Rump, 2004). Because we observed spatial compression in memory for previously viewed environments, the effects of action-specific perception must make contact with the enduring system. This opens the door for new investigations into the influence that action-modified representations may have for navigation, spatial updating, spatial reasoning, and spatial language.

Finally, our findings could have implications for other fields. For instance, investigations into how visual perception differs between phobic versus nonphobic individuals has led to advancements in understanding the etiology of clinical anxiety disorders involving the representation of space (e.g., claustrophobia or acrophobia). For example, individuals with claustrophobia exhibit expanded representations of visual near-body space (Lourenco, Longo, \& Pathman, 2011), and individuals with acrophobia overestimate the distance from a height to the ground (Stefanucci \& Proffitt, 2009). In light of the parallel between the effects observed in the present study and claustrophobic patients' verbalizations of "walls closing in," action-specific effects could provide another useful avenue for understanding potential perceptual mechanisms underlying these disorders. Our results may also have implications for human factors and ergonomics research, which considers the ways in which people interact with manufactured objects. For example, our results suggest an amusing irony of the interior-design process: While we might employ myriad aesthetic tricks to give our living spaces the illusion of seeming bigger, it could be our very use of those spaces that makes them seem smaller.

Acknowledgments ND EPSCoR and NSF Grant No. EPS0814442 provided financial support for this research. The authors thank Christine Allen, Natalie Baldasare, Deborah Cronin, Emily Ehrman, Bryan Golubski, Christopher Held, Alexa Johnson, Shane Kelly, Miles Klimara, Nathan Ochsner, Tim Scully, Sarah Wiesen, and Sylvia Ziejewski for their assistance with data collection.

\section{References}

Bhalla, M., \& Proffitt, D. R. (1999). Visual-motor recalibration in geographical slant perception. Journal of Experimental Psychology: Human Perception and Performance, 25, 1076-1096. doi:10.1037/0096-1523.25.4.1076

Bloesch, E. K., Davoli, C. C., Roth, N., Brockmole, J. R., \& Abrams, R. A. (2012). Watch this! Observed tool use affects perceived distance. Psychonomic Bulletin \& Review, 19, 177-183. doi: 10.3758/s13423-011-0200-z

Davoli, C. C., Brockmole, J. R., \& Witt, J. K. (2012). Compressing perceived distance with remote tool-use: Real, imagined, and remembered. Journal of Experimental Psychology: Human Perception and Performance, 38, 80-89.

Durgin, F. H., Baird, J. A., Greenburg, M., Russell, R., Shaughnessy, K., \& Waymouth, S. (2009). Who is being deceived? The experimental demands of wearing a backpack. Psychonomic Bulletin \& Review, 16, 964-969. doi:10.3758/PBR.16.5.964

Linkenauger, S. A., Witt, J. K., Bakdash, J. Z., Stefanucci, J. K., \& Proffitt, D. R. (2009). Asymmetrical body perception: A possible role for neural body representations. Psychological Science, 20, 1373-1380.

Lourenco, S. F., Longo, M. R., \& Pathman, T. (2011). Near space and its relation to claustrophobic fear. Cognition, 119, 448-453.

Mou, W., McNamara, T. P., Valiquette, C. M., \& Rump, B. (2004). Allocentric and egocentric updating of spatial memories. Journal of Experimental Psychology: Learning, Memory, and Cognition, 30, 142-157. doi:10.1037/0278-7393.30.1.142 
Proffitt, D. R. (2006). Embodied perception and the economy of action. Perspectives on Psychological Science, 1, 110-122.

Shelton, A. L., \& McNamara, T. P. (1997). Multiple views of spatial memory. Psychonomic Bulletin \& Review, 4, 102-106. doi:10.3758/BF03210780

Simons, D. J., \& Wang, R. F. (1998). Perceiving real-world viewpoint changes. Psychological Science, 9, 315-320. doi:10.1111/14679280.00062

Stefanucci, J. K., \& Proffitt, D. R. (2009). The roles of altitude and fear in the perception of height. Journal of Experimental Psychology: Human Perception and Performance, 35, 424-438.

Wang, R. F., \& Simons, D. J. (1999). Active and passive scene recognition across views. Cognition, 70, 191-210.

Wang, R. F., \& Spelke, E. S. (2000). Updating egocentric representations in human navigation. Cognition, 77, 215-250.

Witt, J. K. (2011). Action's effect on perception. Current Directions in Psychological Science, 20, 201-206.
Witt, J. K., \& Dorsch, T. (2009). Kicking to bigger uprights: Field goal kicking performance influences perceived size. Perception, 38, $1328-1340$.

Witt, J. K., Linkenauger, S. A., Bakdash, J. Z., Augustyn, J. A., Cook, A. S., \& Proffitt, D. R. (2009). The long road of pain: Chronic pain increases perceived distance. Experimental Brain Research, 192, 145-148.

Witt, J. K., \& Proffitt, D. R. (2008). Action-specific influences on distance perception: A role for motor simulation. Journal of Experimental Psychology: Human Perception and Performance, 34, 1479-1492.

Witt, J. K., Proffitt, D. R., \& Epstein, W. (2005). Tool use affects perceived distance but only when you intend to use it. Journal of Experimental Psychology: Human Perception and Performance, 31, 880-888.

Witt, J. K., \& Sugovic, M. (2010). Performance and ease influence perceived speed. Perception, 39, 1341-1353. 